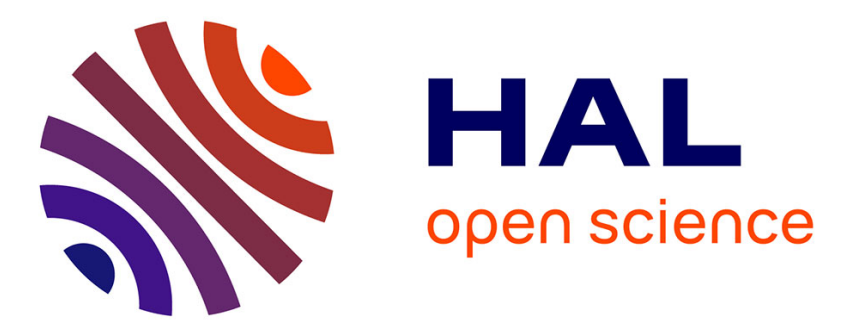

\title{
Application de la diffractométrie des rayons X à l'étude électrochimique de la corrosion de l'acier au carbone en milieu géothermal réel et traité
}

M. Amalhay, I. Ignatiadis

\section{- To cite this version:}

M. Amalhay, I. Ignatiadis. Application de la diffractométrie des rayons X à l'étude électrochimique de la corrosion de l'acier au carbone en milieu géothermal réel et traité. Journal de Physique IV Proceedings, 1996, 06 (C4), pp.C4-913-C4-920. 10.1051/jp4:1996488 • jpa-00254371

\section{HAL Id: jpa-00254371 https://hal.science/jpa-00254371}

Submitted on 1 Jan 1996

HAL is a multi-disciplinary open access archive for the deposit and dissemination of scientific research documents, whether they are published or not. The documents may come from teaching and research institutions in France or abroad, or from public or private research centers.
L'archive ouverte pluridisciplinaire HAL, est destinée au dépôt et à la diffusion de documents scientifiques de niveau recherche, publiés ou non, émanant des établissements d'enseignement et de recherche français ou étrangers, des laboratoires publics ou privés. 


\title{
Application de la diffractométrie des rayons $X$ à l'étude électrochimique de la corrosion de l'acier au carbone en milieu géothermal réel et traité
}

\author{
M. Amalhay et I. Ignatiadis
}

$B R G M$, Direction de la Recherche, Département Géomatériaux et Géoprocédés, 3 avenue C. Guillemin, BP. 6009, 45060 Orléans cedex 2, France

\begin{abstract}
Résumé : Les installations géothermiques exploitant l'aquifère du Dogger du Bassin Parisien connaissent des problèmes de corrosion-dépôt et colmatage dans leurs tubages constitués d'acier au carbone, à cause de la corrosivité des eaux géothermales. Ces eaux constituent un milieu anaérobie caractérisé principalement par la présence de $\mathrm{Cl}^{-}, \mathrm{H}_{2} \mathrm{~S}, \mathrm{SO}_{4}{ }^{2-}$ and $\mathrm{HCO}_{3}{ }^{-}$. Les dépôts de corrosion créés dans ces conditions occasionnent des pertes de charges importantes dans les tubages, ainsi que des corrosions localisées qui peuvent aboutir rapidement au percement des tubages. Pour comprendre l'effet des dépôts de corrosion sur la poursuite de la corrosion de l'acier au carbone des puits géothermiques, des analyses aux rayons $\mathrm{X}$ des dépôts, formés sur l'acier au carbone durant son exposition à un fluide géothermal réel ou traité, ont été entreprises. D'autres techniques spectroscopiques et électrochimiques ont été utilisées pour suivre le comportement de l'acier au carbone au fur et à mesure de la cristallisation des dépôts de corrosion. Des inhibiteurs de corrosion et de croissance cristallines ont été testés au cours de cette étude pour comparer leurs efficacités dans le réduction de la vitesse de corrosion et de la formation des dépôts. En conjuguant les résultats des mesures électrochimiques et les analyses minéralogiques des dépôts de corrosion, nous avons montré que la cristallisation du sulfure de fer amorphe en mackinawite s'accompagne d'une augmentation de la vitesse de corrosion due au transfert des sites cathodiques de la surface du métal à la surface des dépôts. Les inhibiteurs testés réduisent significativement la vitesse de corrosion de l'acier et retardent la cristallisation du sulfure de fer amorphe en mackinawite.
\end{abstract}

\begin{abstract}
Geothermal installations exploiting the Dogger aquifer in the Paris Basin encounter operational difficulties in the form of corrosion of carbon steel well casings, scaling and clogging, due essentially to the corrosivity of geothermal waters. These waters form an anaerobic medium characterized by the presence of $\mathrm{Cl}^{-}$, $\mathrm{H}_{2} \mathrm{~S}, \mathrm{SO}_{4}{ }^{2-}$ and $\mathrm{HCO}_{3}{ }^{-}$. The scale deposited under these conditions causes important head loss in casing as well as localized corrosion that can rapidly lead to perforation of the casing. X-ray analyses of deposits formed on carbon steel during its exposition to an actual or treated geothermal fluid have been undertaken in order to understand the effect of scale on the corrosion behaviour of carbon steel in geothermal wells. Other spectroscopic and electrochemical techniques were used to monitor the behaviour of carbon steel during the crystallization of corrosion deposits. Corrosion and crystal growth inhibitors were tested during the course of this study in order to compare their efficiency in reducting the corrosion rate and the formation of deposits. By combining results of electrochemical measurements and mineralogical analyses of the scale, we have shown that the crystallization of amorphous iron sulfide into mackinawite is accompanied by an increase in the corrosion rate due to the transfer of cathodic sites on the metal at the surface of the scale. All of the inhibitors tested significantly reduce the corrosion rate of the carbon steel and delay the crystallisation of mackinawite from amorphous iron sulphide.
\end{abstract}

\section{INTRODUCTION}

Les eaux de l'aquifère du Dogger du Bassin parisien (Jurassique moyen), exploitées actuellement par une quarantaine de puits géothermiques constituent un milieu anaérobie et temoignent d'un environnement réducteur caractérisé par une minéralisation élevée et la présence de gaz dissous, parmi lesquels $\mathrm{l}^{\prime} \mathrm{H}_{2} \mathrm{~S}$ et le $\mathrm{CO}_{2}$. Les principales caractéristiques de ces eaux, variant selon la localisation des puits, sont les suivantes : température de 47 à $85^{\circ} \mathrm{C}, \mathrm{pH}$ 
de 6,1 à 6,5 , Eh de l'ordre de -100 à $0 \mathrm{mV} / \mathrm{ENH}$, teneur en sels totaux dissous de 6 à $35 \mathrm{~g} / 1$ dont une grande partie en $\mathrm{Cl},\left[\mathrm{CO}_{2} / \mathrm{HCO}_{3}^{-}\right]$de 250 à $600 \mathrm{mg} . \mathrm{l}^{-1}(3-5 \mathrm{mM}),\left[\mathrm{SO}_{4}{ }^{2-}\right]$ de 300 à $1200 \mathrm{mg} \cdot \mathrm{l}^{-1}(0,025-0,125 \mathrm{M}),\left[\mathrm{H}_{2} \mathrm{~S} / \mathrm{HS}^{-}\right]$de 0 à $100 \mathrm{mg} \cdot \mathrm{l}^{-1}(0-3 \mathrm{mM})$. La concentration en $\mathrm{H}_{2} \mathrm{~S} / \mathrm{HS}^{-}$varie avec le temps et le débit d'exploitation [1]. Les caractéristiques de ces eaux le rendent corrosives pour les tubages des puits qui sont en majorité constitués d'acier au carbone $[2,3]$. Au niveau des installations géothermiques le risque lié à cette corrosivité engendrait les problèmes suivants :

- une durée de vie des puits souvent inférieure à la moitié de son estimation,

- le percement des tubages en acier des puits avec contamination possible d'aquifères intermédiaires constituant, pour la plupart, des réserves d'eau potable,

- la formation de dépôts sur les parois internes des tubages susceptibles de colmater les puits de production et/ou de réinjection, ce qui entraîne des pertes de débit significatives.

Du point de vue historique, c'est cette dernière conséquence qui a été observée la première. Les difficultés d'exploitation sur certains des puits sont apparues avec une baisse des caractéristiques hydrauliques, colmatage partiel des puits de production et/ou de réinjection, encrassement des échangeurs thermiques. Les nombreuses opérations de réhabilitation des puits effectuées ont permis de corréler les problèmes de l'exploitation à la présence de dépôts. Ces travaux ont montré que les puits produisant un fluide de forte teneur en $\mathrm{H}_{2} \mathrm{~S} / \mathrm{HS}^{-}$sont les plus touchés par la corrosion et le colmatage. Des dépôts de sulfures de fer se forment à partir du $\mathrm{H}_{2} \mathrm{~S} / \mathrm{HS}^{-}$présent dans le fluide et le $\mathrm{Fe}^{2+}$ issu principalement de la corrosion des tubages. Des mesures de vitesse de corrosion, effectuées par différentes méthodes sur des puits sans traitement par inhibiteur de corrosion, donnent fréquemment des valeurs de l'ordre de $1 \mathrm{~mm} / \mathrm{an}$. Les dépôts sont constitués à près de $70 \%$ de sulfures de fer : mackinawite $\left(\mathrm{Fe}_{1+\mathrm{x}} \mathrm{S}\right.$ ), pyrite $\left(\mathrm{FeS}_{2}\right)$ et pyrrhotite $\left(\mathrm{FeS}_{1+\mathrm{x}}\right)$. Sont présents en faibles quantités les carbonates de fer $\left(\mathrm{FeCO}_{3}\right)$, les hydroxydes de fer $\mathrm{Fe}(\mathrm{OH})_{2}$, les oxyhydroxydes de fer $(\mathrm{FeOOH})$ et hydroxychlorures de fer $\left(\mathrm{Fe}_{2}(\mathrm{OH})_{3} \mathrm{Cl}\right)$, ainsi que la calcite $\left(\mathrm{CaCO}_{3}\right)$ principalement dans les échantillons de fond (entre 10 et $20 \%$ ). La stoechiométrie des sulfures de fer évolue avec la profondeur (mackinawite en surface et pyrite en fond de puits) et au sein du dépôt (mackinawite côté tubage et pyrite côté fluide). La chronologie des dépôts est complexe et semble résulter de plusieurs phénomènes statiques et dynamiques (précipitation, transport, vieillissement) [4]; mais elle peut refléter également les conditions d'exploitation [5].

Pour pallier à ces problèmes, la corrosion des tubages et le colmatage des puits, deix niveaux de réflexion se sont développés : d'une part, la prévention des risques de corrosion par l'utilisation de matériaux nouveaux (revêtement anti-corrosion, aciers spéciaux, échangeurs en titane,...), d'autre part, la protection des ouvrages existants par traitement du fluide.

La mise en oeuvre de traitements anti-corrosion par l'injection au fond du puits de production d'agents tensio-actifs cationiques (inhibiteurs de corrosion efficaces à des teneurs très faibles, $2,5 \mathrm{mg} \cdot \mathrm{l}^{-1}$ en moyenne et à des coûts acceptables) a permis de résoudre la plus grande partie des problèmes de corrosion/dépôts identifiés. Toutefois, il apparaît aujourd'hui sur certains sites des problèmes nouveaux. Il s'agit principalement :

- des phénomènes de déposition qui persistent et de corrosion sous-dépôt, qui dans la majorité des cas prend la forme de piqûres [4-5],

- de l'inadaptation des inhibiteurs à la spécificité de chaque fluide (pH, teneurs en $\mathrm{HS}^{-}, \mathrm{Cl}^{-}$, $\mathrm{HCO}_{3}^{-}$),

- de la croissance de l'activité bactérienne dans certains puits (croissance de teneurs en $\mathrm{HS}^{-}$).

Cet article présente une étude relative aux deux premiers problèmes cités. Elle est basé sur la détermination minéralogique des phases présentes dans les dépôts obtenus sur les échantillons d'acier au carbone suivis électrochimiquement en milieu géothermal réel ou traité par inhibiteur de corrosion. 


\section{MATERIEL ET METHODES}

Pour approcher le comportement réel des tubages des puits cette étude a été menée sur un site géothermique non traité par inhibiteur. La composition physico-chimique du fluide de ce site est représentative des sites du sud du Bassin parisien $\left(60^{\circ} \mathrm{C} ; \mathrm{pH} 6,2 ;\left[\mathrm{Cl}^{-}\right] 7,2\right.$ g..$^{-1} ;\left[\mathrm{H}_{2} \mathrm{~S}, \mathrm{HS}^{-}\right]$ 14.mg.1.-1; $\left[\mathrm{CO}_{2}, \mathrm{HCO}_{3}^{-}\right] 348{\mathrm{mg} . l^{-1}}^{-1}$.

Des électrodes de travail, fixes et rotatives (1000 rpm), de surfaces respectives, 2 et $1 \mathrm{~cm}^{2}$ ont été utilisées. Elles sont fabriqués dans l'acier au carbone dont la composition moyenne de l'acier est la suivante: C: $0,42 \%$; $\mathrm{Mn}: 1,08 \%$; Mo: $0,014 \%$; $\mathrm{Si}: 0,34 \%$; $\mathrm{Ni}: 0,092 \%$; $\mathrm{Cr}$ : $0,100 \%$ et $\mathrm{S}: 0,004 \%$. Des électrodes au calomel saturé sont utilisées comme électrodes de référence. Des cylindres maillés, de grande surface, en platine iridié (Pt: 90\% ; Ir: 10\%) sont utilisés comme contre-électrodes.

Le fluide, prélevé en dérivation par un piquage en tête du puits de production, est acheminé sous pression vers deux cellules par deux tuyaux calorifugés. Le débit est maintenu constant à $30 \mathrm{l} / \mathrm{h}$. La pression dans les réacteurs est de l'ordre de $1 \mathrm{~atm}$. Avant d'arriver aux réacteurs, le fluide passe par des mélangeurs prévus dans le cas d'ajout d'inhibiteurs de corrosion à la solution. Pour l'injection de ces additifs, des pompes de grande précision ont été utilisées.

Durant les expériences, les paramètres $\mathrm{pH}, \mathrm{Eh}$, température et concentration du fluide en inhibiteur, ont été suivis. Pour le suivi électrochimique des électrodes deux potentiostatsgalvanostats (EG\&G Parc, modèle 273A) et un amplificateur à détection synchrone EG\&G Parc, Lock-In Amplifier modèle 5210, pilotés par ordinateur, ont été utilisés.

Différents procédés expérimentaux ont été utilisés pour évaluer l'efficacité des six inhibiteurs, dont trois sont actuellement utilisés en géothermie (Solamine 129 (SEPPIC, Paris, France), Norust $491^{\circledR}$ (CECA, La Défense, France), Aquaprox MDC1300® (PROTEX, Levallois, France), alors que les trois autres molécules ou formulations (appelées Amine Cyclique, Amine Soluble et Amine Grasse) sont non commercialisées. Les inhibiteurs sont injectés soit avant et au cours de l'immersion des électrodes à une concentration de $10 \mathrm{mg} . \mathrm{l}^{-1}$, soit un ajout de 2,5 puis $5 \mathrm{mg} .1^{-1}$ pendant $10 \mathrm{~h}$ après une période d'immersion sans traitement de l'ordre de $12 \mathrm{~h}$.

Les dépôts présents sur les échantillons d'acier sont protégés sur le site afin de limiter leur altération (oxydation en particulier) avant les analyses appropriées. Pour les analyses par diffractométrie des rayons $\mathrm{X}$, les produits de corrosion sont broyés, mélangés et pastillés sous alcool, puis séchés sous vide et ensuite analysés à l'aide d'un appareillage Philips (Rayonnement $\mathrm{Co} \mathrm{K \alpha}$ avec monochromateur arrière en graphite, sensibilité $2000 \mathrm{c} / \mathrm{s}$, domaine d'exploration de spectres: 4 à $84^{\circ} 2 \theta$ ). Pour les analyses de surface par MEB, microscopie métallographique et microsonde électronique, les échantillons (coupons ou produits de corrosion) sont enrobés de résine pour conserver les produits de corrosion, puis polis avec des papiers abrasifs à rugosité décroissante jusqu'à la finition à la pâte diamantée $(0,1 \mu \mathrm{m})$.

\section{RESULTATS}

\subsection{Comportement de l'acier en absence d'inhibiteur}

Le comportement de l'acier dans un tel milieu était encore mal connu. La protectivité naturelle de la surface de l'acier en présence des dépôts l'est encore moins. Les analyses des dépôts recueillis à la surface d'aciers immergés dans l'eau géothermal pendant de longues durées révèlent l'existence parmi les dépôts de plusieurs phases de sulfures de fer $[4,5]$. Depuis bien longtemps, des travaux ont montré que les potentiels normaux de ces différentes phases ainsi que leur protectivité sont très différents [7-8]. 
Afin de mieux comprendre ces mécanismes et de discerner l'action des inhibiteurs de corrosion sur le comportement de l'acier au carbone dans l'eau géothermale, des suivis du comportement électrochimique de l'acier ont été effectués sur des électrodes statiques et rotatives pendant de longues durées d'immersion sans inhibiteur.

\subsubsection{Evolution du potentiel libre de corrosion, $E_{\text {cor }}$}

Après leur immersion dans l'eau géothermale, les électrodes voient leur potentiel de corrosion augmenter en fonction du temps (Fig. 1). Les périodes d'évolution du $\mathrm{E}_{\mathrm{cor}}$ sont fonction de la vitesse de rotation de l'électrode. La surface de l'électrode rotative évolue beaucoup plus vite que celle de l'électrode statique. Cette différence se manifeste dès les premières minutes d'immersion et s'accentue au cours du temps pour atteindre une valeur moyenne d'environ 100 $\mathrm{mV}$ après les 20 premières heures d'immersion.

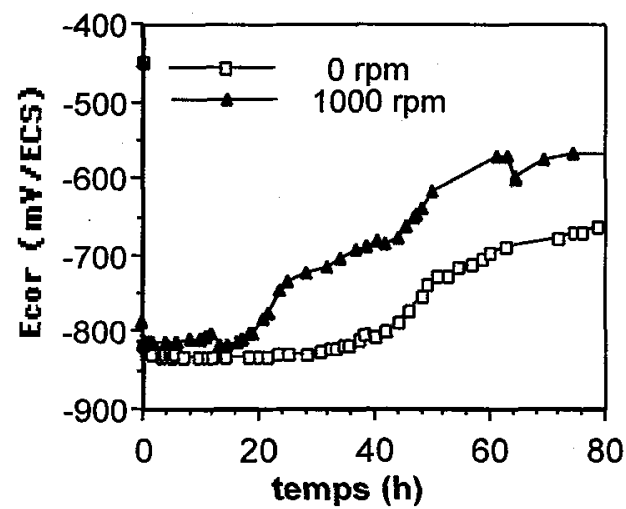

Figure 1: Evolution du potentiel libre de corrosion de l'acier au carbone en fonction de la vitesse de rotation de l'électrode (statique et rotative) et du temps d'immersion dans l'eau géothermale non traitée.

Evolution of the open circuit potential of carbon steel (as a function of rotation speed of the electrode (stationary and rotating) and immersion time in geothermal water without treatment.

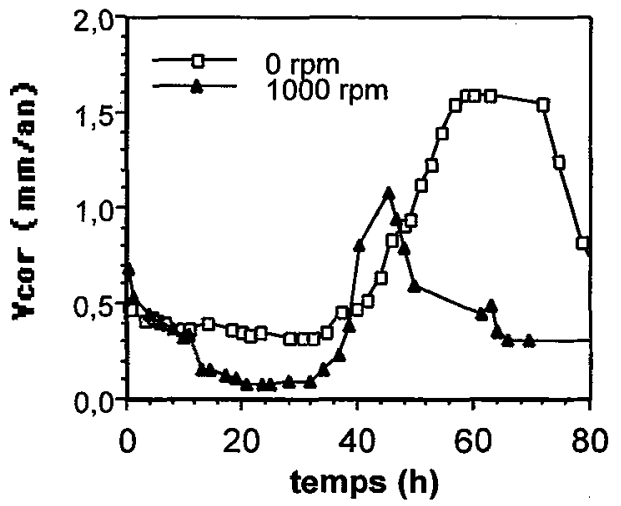

Figure 2: Evolution de la vitesse de corrosion de l'acier au carbone en fonction de la vitesse de rotation de l'électrode (statique et rotative) et du temps d'immersion dans l'eau géothermale non traitée.

Evolution of the corrosion rate of carbon steel as a function of rotation speed of the electrode (stationary and rotating) and immersion time in geothermal water without treatment.

\subsubsection{Evolution de la vitesse de corrosion, $V_{\text {cor }}$}

L'allure des courbes de polarisation ainsi que les diagrammes d'impédance obtenus durant ces essais, montrent que la cinétique de corrosion dans ces conditions est régie par transfert de charges. Les vitesses de corrosion ont donc été calculées à partir des mesures de résistances de polarisation et des pentes cathodiques et anodiques de Tafel, en appliquant l'équation de Stern et Geary, [9]. Sur les courbes représentant les vitesses de corrosion en fonction du temps d'immersion des électrodes statiques et rotatives (Fig. 2), il est possible de distinguer quatre grandes étapes qui traduisent toutes des comportements différents de l'acier :

Une première étape, qui commence dès l'immersion des échantillons, où la vitesse de corrosion diminue rapidement. Cette diminution, plus marquée dans le cas des électrodes 
rotatives, est due à la formation d'un dépôt protecteur. Le fait que cette diminution s'effectue à potentiel constant (Fig. 1) traduit une diminution des activités anodique et cathodique.

Une deuxième étape, plus évidente et plus lente dans le cas de l'électrode statique, montre une stabilisation de la vitesse de corrosion. Dans le cas de l'électrode rotative cette stabilisation s'accompagne d'une augmentation du potentiel libre de corrosion. L'analyse des courbes de polarisation montre une augmentation de l'activité cathodique.

Une troisième étape, où la vitesse de corrosion augmente malgré l'anoblissement progressif des électrodes, jusqu'à des valeurs qui dépassent largement la vitesse de corrosion initiale. L'analyse des courbes de polarisation, tracées au cours de cette période, montre une augmentation significative des activités anodique et cathodique.

Une quatrième étape durant laquelle la vitesse de corrosion commence à décroître pour atteindre une valeur moyenne, qui dépend des conditions hydrodynamiques.

Il est à remarquer que ces quatre étapes sont plus courtes sur les électrodes rotatives (Fig. 2).

Pour comprendre ces changements dans le comportement de la surface de lacier durant ces 50 premières heures d'immersion, plusieurs porte-coupons de corrosion ont été placés dans les mêmes conditions physico-chimiques que précédemment pendant différents temps d'immersion $(0,5,10,5,20,40,70$ et 140 heures $)$. Sur chaque porte-coupon ont été fixées deux électrodes de $1 \mathrm{~cm}^{2}$ de surface. Aucune perturbation électrique externe n'a été appliquée à ces coupons de corrosion au cours de leur immersion. Les analyses par diffractométrie des rayons $\mathrm{X}$ des dépôts de corrosion, recueillis à la surface des coupons, ont montré une évolution des phases des dépôts de sulfures de fer. En effet, les dépôts recueillis après $20 \mathrm{~h}$ d'immersion sont en majorité amorphes (Fig. 3a). La mackinawite bien cristallisée commence à apparaître après $40 \mathrm{~h}$ d'immersion, ce qui coïncide avec l'augmentation des vitesses de corrosion mesurées par les techniques électrochimiques (3ième étape). Après 70 et $140 \mathrm{~h}$ d'immersion (4ième étape), les dépôts sont constitués en grande majorité de mackinawite très bien cristallisée (Fig. 3b).

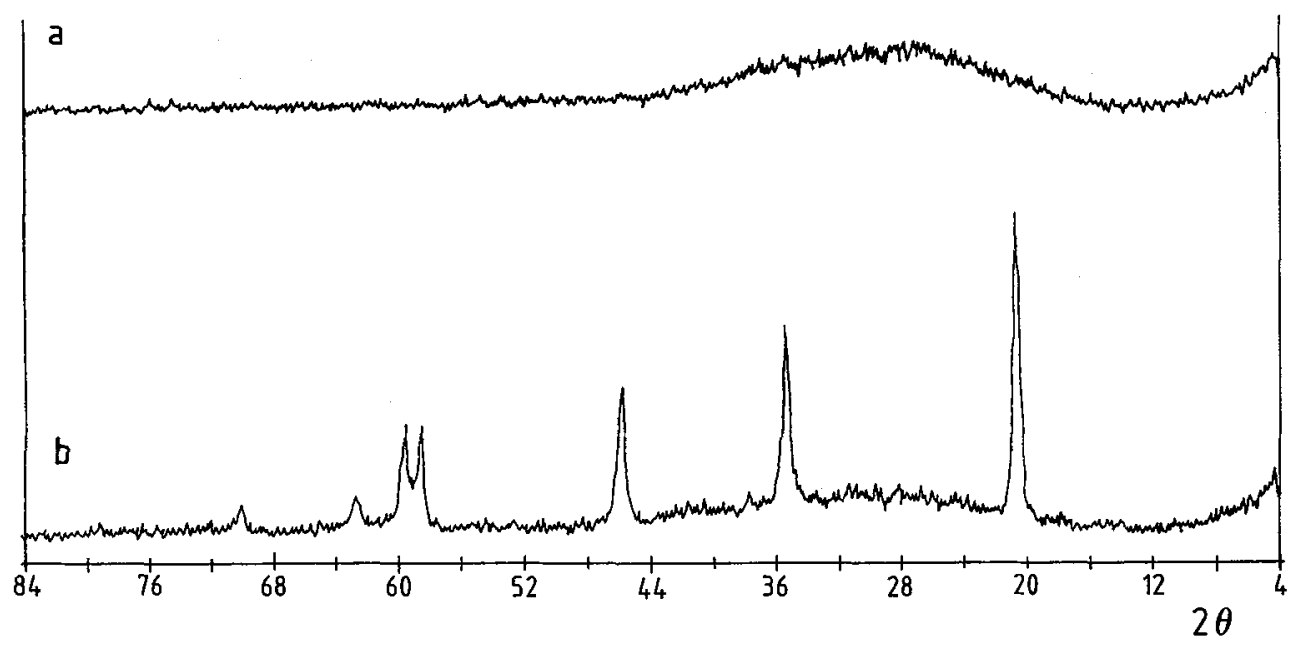

Figure 3: Diffractogrammes X du dépôt obtenu sur l'acier au carbone a) après 20 heures d'immersion b) après 60 heures d'immersion dans le fluide de Melun l'Almont non traité par inhibiteur.

$\mathrm{X}$-ray difractograms of scale on carbon steel a) after $20 \mathrm{~h}, \mathrm{~b}$ ) after $60 \mathrm{~h}$ of immersion in the Melun l'Almont fluid, not treated with inhibitor. 
L'augmentation de la vitesse de corrosion dans la troisième étape peut être expliquée : le dépôt formé initialement sur l'acier est le sulfure de fer amorphe qui est partiellement protecteur. Ce dépôt commence à cristalliser après un certain temps d'immersion de l'ordre de 20 à 35 heures, Ce temps du début de cristallisation dépend du régime hydrodynamique de l'électrode. Cette cristallisation du dépôt s'accompagne, d'une part d'une augmentation de porosité et de défauts dans le dépôt et, d'autre part, d'un transfert des sites cathodiques de la surface du métal à la surface du dépôt. La diminution de la vitesse de corrosion lors de la quatrième étape est certainement due au colmatage, par de nouveaux précipités amorphes, des défauts crées dans le dépôt lors de la cristallisation du sulfure de fer amorphe en mackinawite.

\subsection{Comportement de l'acier en présence d'inhibiteur de corrosion}

\subsubsection{Ajout préalable d'inhibiteur}

Le suivi du $\mathrm{E}_{\text {cor }}$ de l'acier, juste après son immersion dans l'eau géothermale traitée dès le début avec les différents inhibiteurs à $10 \mathrm{mg} .1^{-1}$, montre un anoblissement des électrodes. Le $\mathrm{E}_{\text {cor }}$ augmente de 50 à $200 \mathrm{mV}$ durant les 20 premières minutes d'immersion. Cet anoblissement du métal est dû à l'adsorption des inhibiteurs sur la surface de l'acier et par suite à une réduction de la vitesse de corrosion. Ainsi, les variations du $\mathrm{E}_{\text {cor }}$ des échantillons en présence de chaque inhibiteur peuvent correspondre à sa vitesse d'adsorption sur la surface du métal. Les Amines Grasse et Soluble, ainsi que le Norust 491 ont la vitesse d'adsorption la plus rapide.

\subsubsection{Ajout d'inhibiteur après une période d'immersion sans traitement}

L'efficacité des inhibiteurs testés est évaluée pendant des périodes de traitement à 2,5 et 5 mg. $1^{-1}$ après une première phase de $12 \mathrm{~h}$ d'immersion sans traitement. L'injection des inhibiteurs, même après la formation préalable des dépôts de corrosion, a provoqué des réponses plus au moins immédiates de l'acier. Ces réponses ont été caractérisées par une augmentation des potentiels libres de corrosion, une augmentation des résistances de polarisation et une baisse de lactivité anodique. Il en a résulté une diminution de la vitesse de corrosion des électrodes. Sur la figure 4 sont comparées les vitesses de corrosion de l'acier au carbone en présence et en absence d'inhibiteur. On remarque que l'ajout de la Solamine provoque une forte baisse de la vitesse de corrosion. De plus, l'augmentation de la vitesse de corrosion mesurée après les 35 h d'immersion, dans le fluide non traité, disparaît. Par ailleurs, l'évolution de la vitesse de corrosion d'une électrode statique a été suivie en maintenant la concentration de l'Amine Soluble à $2,5 \mathrm{mg} .1^{-1}$. Bien que la réduction de la vitesse de corrosion au début du traitement soit comparable à celle obtenue avec la Solamine, l'allure de la courbe se rapproche de celle des électrodes sans traitement : l'accroissement de la vitesse de corrosion, après $35 \mathrm{~h}$ d'immersion, se manifeste encore mais de façon plus atténuée (Fig. 5).

Les diffractogrammes relatifs à la faible quantité de dépôts de sulfures de fer formés sur l'acier au carbone en présence d'inhibiteur de corrosion (Solamine 129) après 60 heures d'immersion ont une allure comparable à celle de la figure $3 \mathrm{a}$. Ceci demontre qu'ils sont restés à l'état amorphe. Ce changement dans le comportement à la corrosion de l'acier en présence de la majorité des inhibiteurs peut être imputé au moins à deux phénomènes : 
- la disparition ou le ralentissement des transformations intrinsèques des dépôts, notamment de la cristallisation du sulfure de fer amorphe en mackinawite. En effet, les analyses minéralogiques des dépôts recueillis à différentes périodes d'immersion montrent qu'en présence des inhibiteurs, les sulfures de fer restent amorphes en majeure partie.

- la réduction de l'effet d'activation des processus cathodiques de corrosion par les dépôts. En effet, les inhibiteurs de corrosion réduisent la réaction cathodique en s'adsorbant sur les dépôts en surface et à l'interphase, bloquant ainsi partiellement les sites cathodiques.

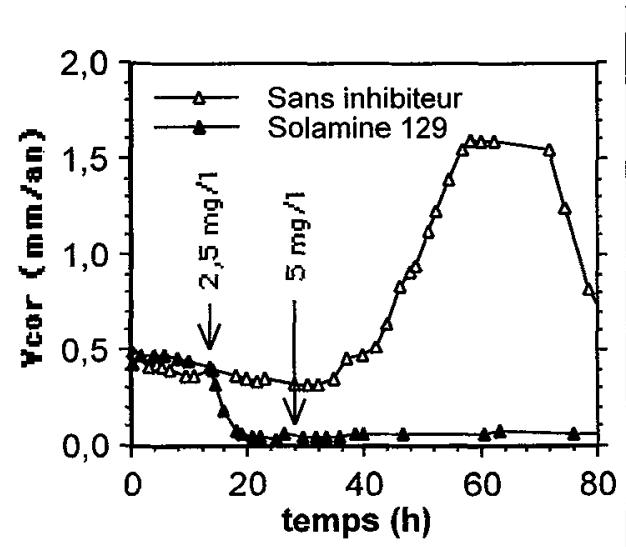

Figure 4: Effet de l'ajout de 2,5 puis $5 \mathrm{mg}^{-1}$ de Solamine sur la vitesse de corrosion de l'acier au carbone (électrode statique) dans l'eau géothermale. Effect of adding 2.5 then $5 \mathrm{mg} \cdot \mathrm{l}^{-1}$ of Solamine on the evolution of the steel corrosion rate (stationary electrode) in geothermal water.

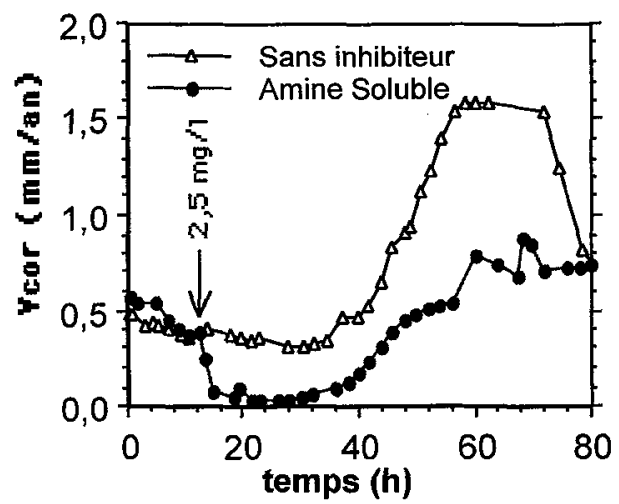

Figure 5: Evolution de la vitesse de corrosion de l'acier au carbone (électrode statique) dans l'eau géothermale traitéé par $2,5 \mathrm{mg} \cdot \mathrm{l}^{-1} \mathrm{~d}$ 'Amine Soluble. Effect of adding $2.5 \mathrm{mg} \cdot \mathrm{l}^{-1}$ of Soluble Amine on the evolution of the steel corrosion rate (stationary electrode) in geothermal water.

\subsection{Comportement de l'acier après l'arrêt du traitement}

En cas d'un arrêt prolongé du traitement, une cristallisation des dépôts de sulfure de fer amorphe en mackinawite peut avoir lieu. Cette transformation s'accompagnera d'une augmentation des défauts dans les dépôts et d'une augmentation de la vitesse de corrosion. Pour évaluer ce risque, le comportement électrochimique à la corrosion des électrodes statiques ainsi qu'une analyse minéralogique des dépôts ont été effectués après l'arrêt de l'injection des inhibiteurs pendant des temps d'immersion allant de 10 à $90 \mathrm{~h}$ d'immersion.

L'arrêt de l'injection des inhibiteurs a provoqué une diminution progressive du potentiel libre de corrosion de l'acier dans l'eau géothermale pendant les premières 10 à $14 \mathrm{~h}$ d'immersion, ainsi qu'une augmentation de la vitesse de corrosion. Ce comportement est donc dû à la désorption des inhibiteurs. L'arrêt pendant $95 \mathrm{~h}$ du traitement par. l'Amine Cyclique a provoqué, après une légère baisse, dans les premières dix heures d'immersion, une augmentation importante du potentiel libre $(123 \mathrm{mV})$ et de la vitesse de corrosion. Ce comportement s'explique par la reprise de la cristallisation du sulfure de fer amorphe et donc l'activation de la réaction cathodique. 


\section{CONCLUSION}

Le comportement électrochimique de l'acier au carbone immergé dans le fluide géothermal réel a été étudié pendant différentes durées en absence et en présence d'inhibiteur de corrosion. La conjugaison des résultats du suivi électrochimique de l'acier au carbone et les analyses minéralogiques des dépôts de corrosion par diffractométrie des rayons $\mathrm{X}$ ont permis de mettre en évidence que :

1) la cristallisation du dépôt de sulfure de fer amorphe en mackinawite a lieu au bout de quelques dizaines d'heures d'immersion. Le temps du début de cristallisation dépend du régime hydrodynamique de l'électrode.

2) la cristallisation s'accompagne d'une augmentation de la vitesse de corrosion due au transfert des sites cathodiques de la surface du métal à la surface des dépôts.

3) les dépôts de sulfures de fer grâce à leur conductivité permettent la poursuite de la corrosionde l'acier au carbone sous le dépôt.

La présence des inhibiteurs, même à $2,5 \mathrm{mg} . \mathrm{l}^{-1}$, provoque un anoblissement de l'acier, une forte augmentation des résistance de polarisation, ainsi qu'une réduction de la vitesse de la cristallisation des dépôts de sulfure de fer amorphe en mackinawite. Ces inhibiteurs réduisent les courants anodiques et cathodiques et sont plus efficaces sur les électrodes statiques que sur les rotatives. L'arrêt de l'injection des inhibiteurs de corrosion dans la solution provoque une augmentation de la vitesse corrosion, une diminution du potentiel libre de corrosion, ainsi qu'une reprise de la cristallisation du sulfure de fer amorphe après une période de désorption d'environ dix heures.

\section{Remerciements}

Cet article est la contribution BRGM N ${ }^{\circ} 95045$. Le travail, effectué dans le cadre du projet S08 de la Direction de Recherche du BRGM, a reçu le soutien financier de la Commission de l'Union Européenne (contrat NoJOU2-CT92-0108).

\section{Références}

[1] Ignatiadis I., "Origins of the increased sulphide concentrations noted in geothermal fluids at production wellheads in the south and east of the Paris basin", Geothermics 94 in Europe, from Research to Development, Orléans 8-9 February 1994 (Les éditions du BRGM, Orléans, France, 1994), pp. 241-248.

[2] Ellis P.E., GRC Transactions 5 (1981) 463-472.

[3] Ignatiadis I., Teton S., GRC Transactions 16 (1991) 357-363.

[4] Amalhay M., Abou Akar A., Ignatiadis I., "Overview of the deposition phenomena in geothermal wells in the Paris Basin", World Geothermal Congress, 1995, Florence, Italy, 1831 May 1995 (International Geothermal Association, Inc., Auckland, New Zeland, 1995, Volumes 1 to 5), 4 pp. 2497-2502.

[5] Amalhay M., Gauthier B., Ignatiadis I., "The influence of some physico-chemical parameters and exploitation conditions on corrosion and scaling in geothermal wells in the Paris Basin", Geothermics 94 in Europe, from Research to Development, Orléans 8-9 February 1994 (Les éditions du BRGM, Orléans, France, 1994), pp. 233-240.

[6] Greco E. C. And Wright W. Corrosion 18 (1961) 119-124.

[7] Hausler R. H., Corrosion Chemistry, ACS Symp. Ser. 89 (1982), 307-317

[8] Smith J.S., Miller J.D., Br. Corros. J. 10, (1975) 136-143.

[9] Stern M., Geary A.L., J. Electrochem. Soc. 104 (1957) 559-563. 